\title{
Reversible Insertion of Carbon Dioxide at Phosphine Sulfonamido PdII-Aryl-Complexes
}

Gregor Voita ${ }^{a}$ Sangeth Jenthraa, Markus Hölscher*a, Thomas Weyhermüller ${ }^{\mathrm{b}}$ and Walter Leitner*a,b

a Institut für Technische und Makromolekulare Chemie, RWTH Aachen University, Worringerweg 2, 52074 Aachen, Germany. E-mail: leitner@itmc.rwth-aachen.de

b Max Planck Institute for Chemical Energy Conversion, Mülheim an der Ruhr, Germany, E-Mail: walter.leitner@cec.mpg.de

\section{Table of Contents}

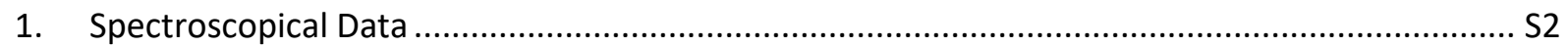

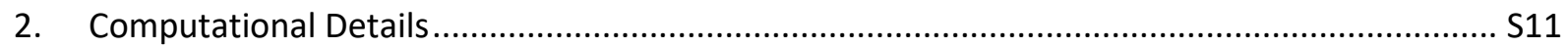

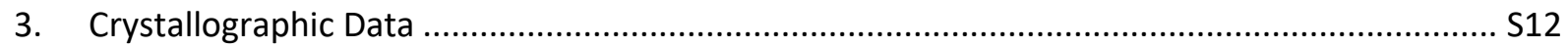

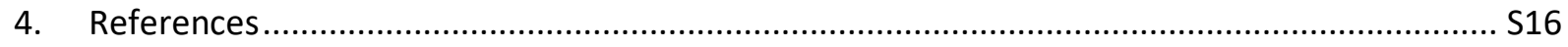




\section{Spectroscopical Data}

\subsection{1-pAn}

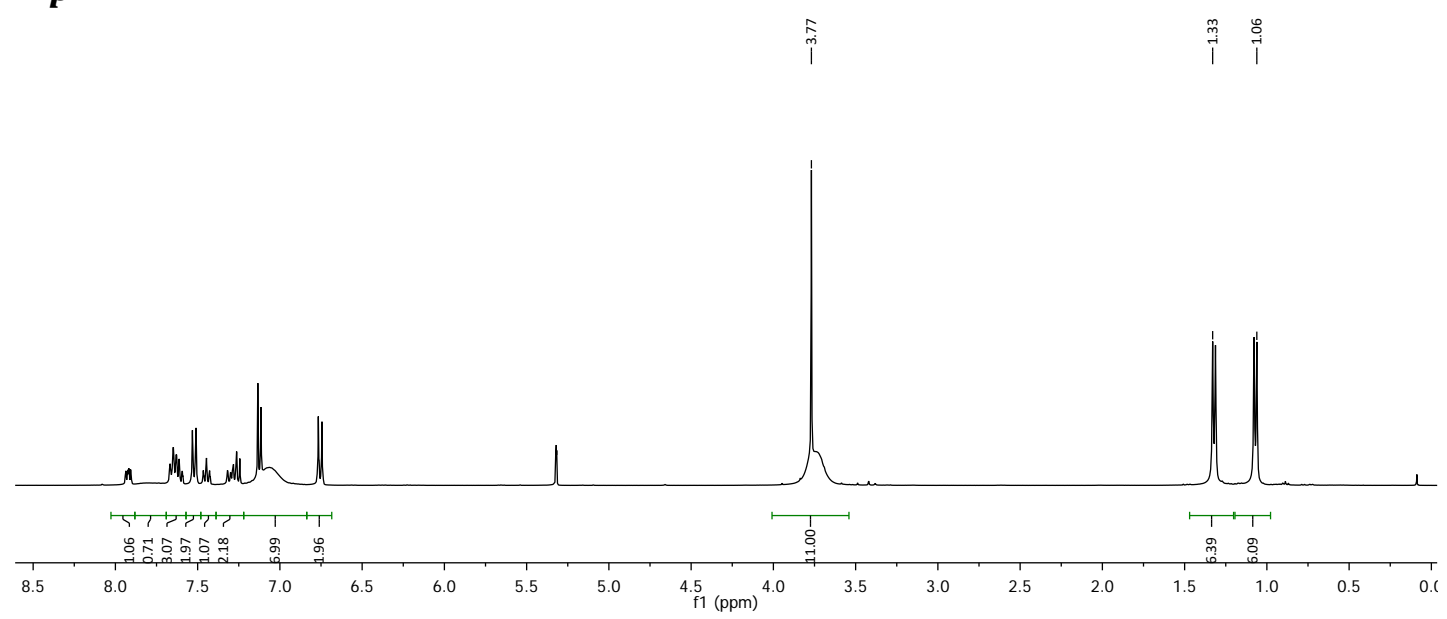

Figure S1: ${ }^{1} \mathrm{H}-\mathrm{NMR}$ spectrum $\left(\mathrm{CD}_{2} \mathrm{Cl}_{2}, 25^{\circ} \mathrm{C}, 400 \mathrm{MHz}\right)$ of 1-pAn.

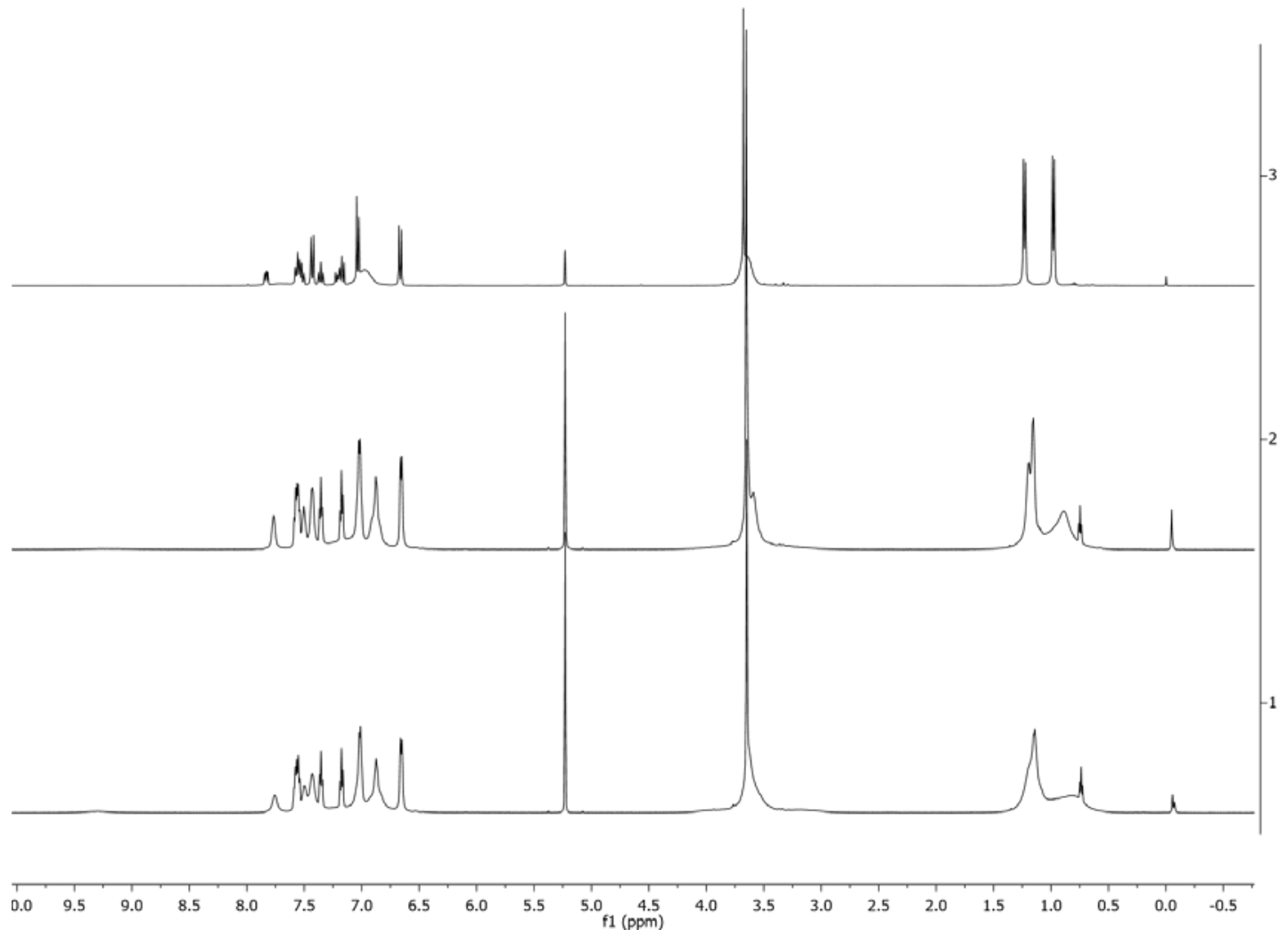

Figure S2: ${ }^{1} \mathrm{H}-\mathrm{NMR}$ spectrum $\left(\mathrm{CD}_{2} \mathrm{Cl}_{2}, 400 \mathrm{MHz}\right)$ of 1-pAn at $25{ }^{\circ} \mathrm{C}(3)$ and ${ }^{1} \mathrm{H}-\mathrm{NMR}$ spectra $\left(\mathrm{CD}_{2} \mathrm{Cl}_{2}\right.$, $600 \mathrm{MHz})$ of 1-pAn at $-30{ }^{\circ} \mathrm{C}(2)$ and $-40{ }^{\circ} \mathrm{C}(1)$. 


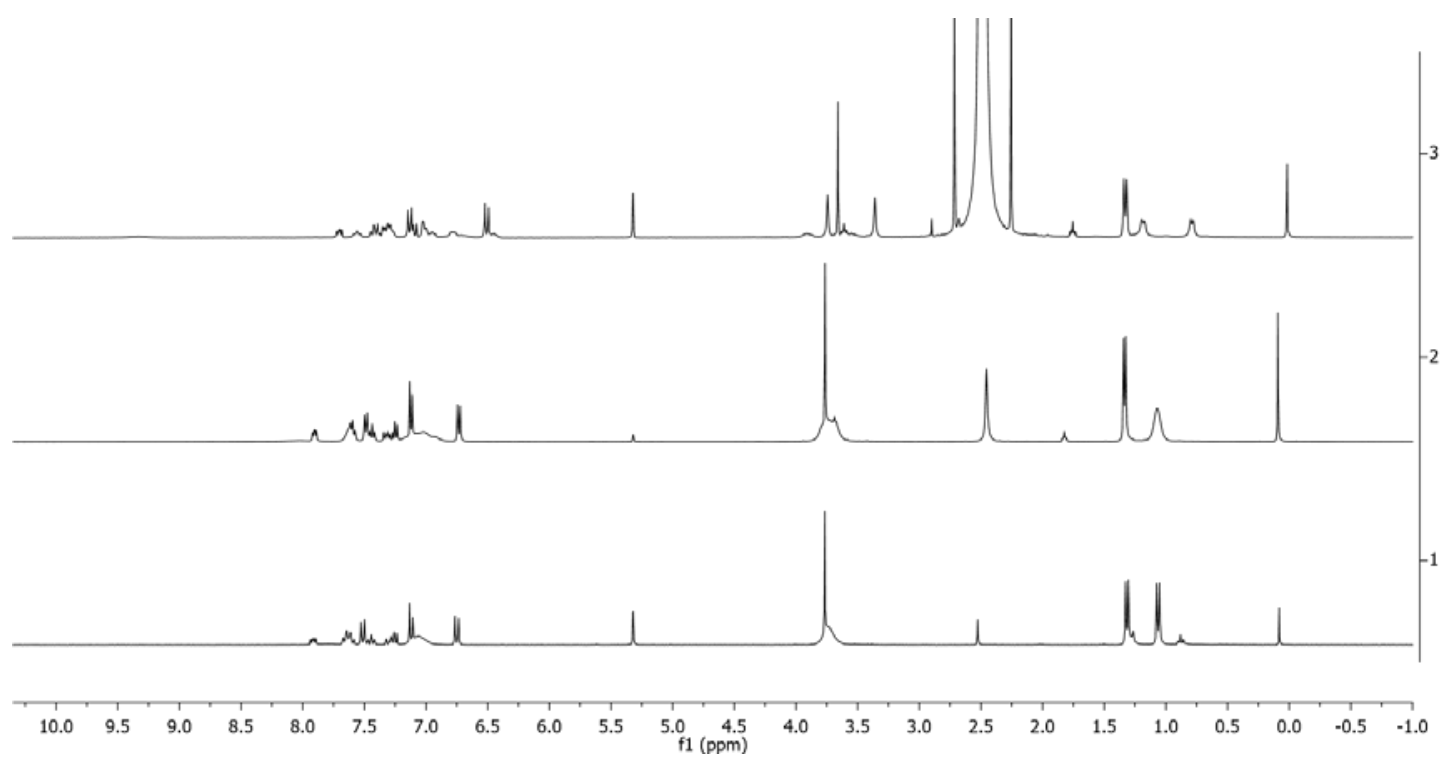

Figure S3: ${ }^{1} \mathrm{H}$-NMR spectra $\left(\mathrm{CD}_{2} \mathrm{Cl}_{2}, 25^{\circ} \mathrm{C}, 300 \mathrm{MHz}\right.$ for 1 and 3, $400 \mathrm{MHz}$ for 2) of 1-pAn after addition of 0.15 eq (1), 0.8 eq (2) and 215 eq (3) of DMSO under partial coordination of DMSO to 1-pAn.

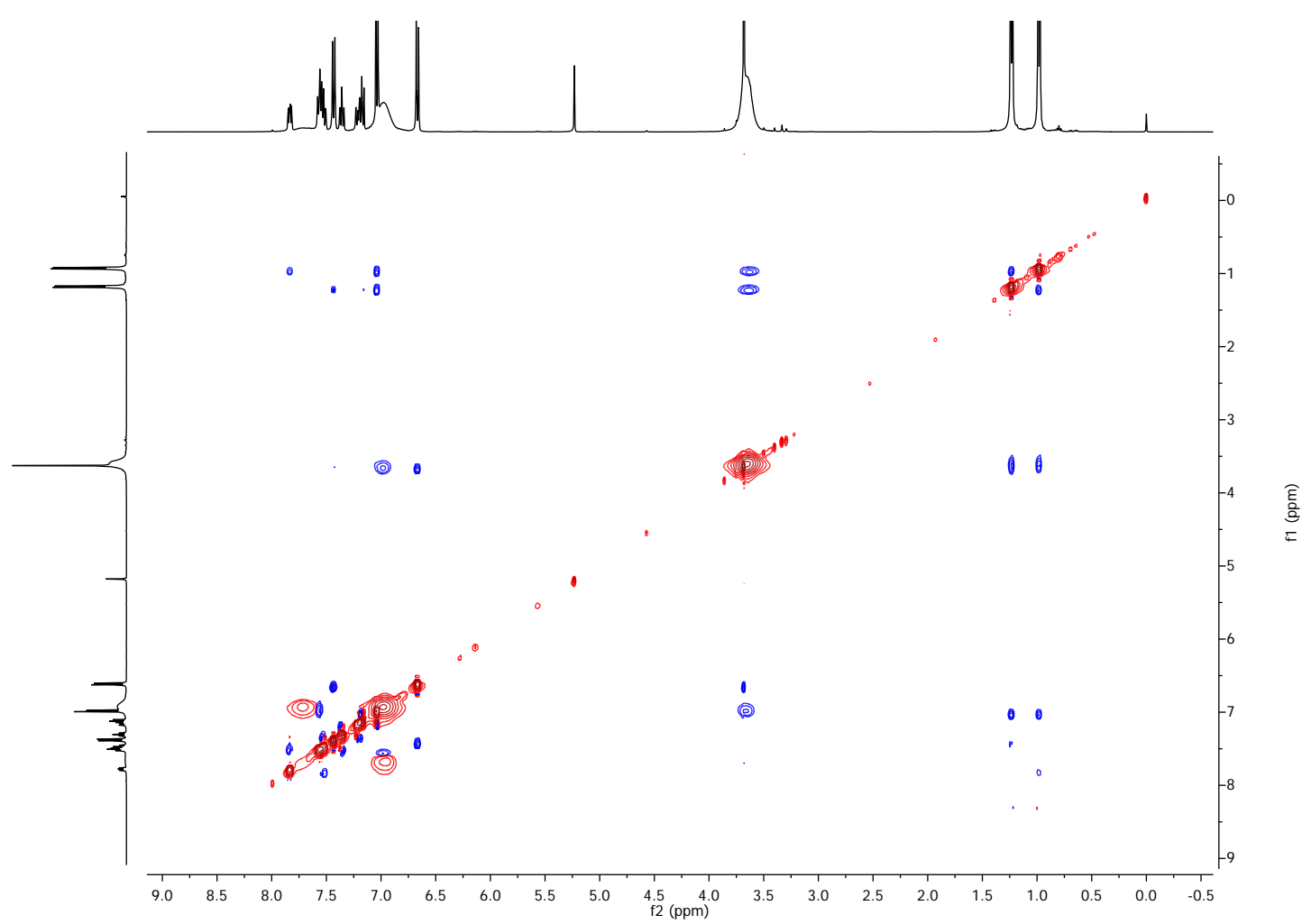

Figure S4: ${ }^{1} \mathrm{H}-1 \mathrm{H}-\mathrm{NOESY}$ spectrum $\left(\mathrm{CD}_{2} \mathrm{Cl}_{2}, 25^{\circ} \mathrm{C}, 400 \mathrm{MHz}, 0.8 \mathrm{~s}\right.$ mixing time $)$ of 1-pAn. 


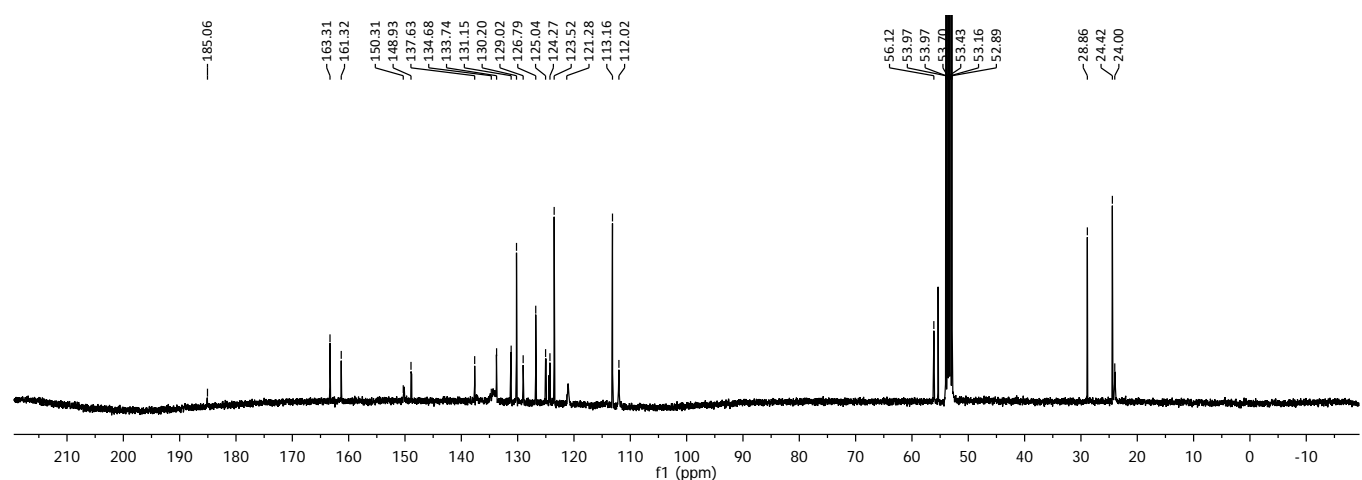

Figure S5: ${ }^{13} \mathrm{C}$-NMR spectrum $\left(\mathrm{CD}_{2} \mathrm{Cl}_{2}, 25^{\circ} \mathrm{C}, 100 \mathrm{MHz}\right)$ of 1-pAn.

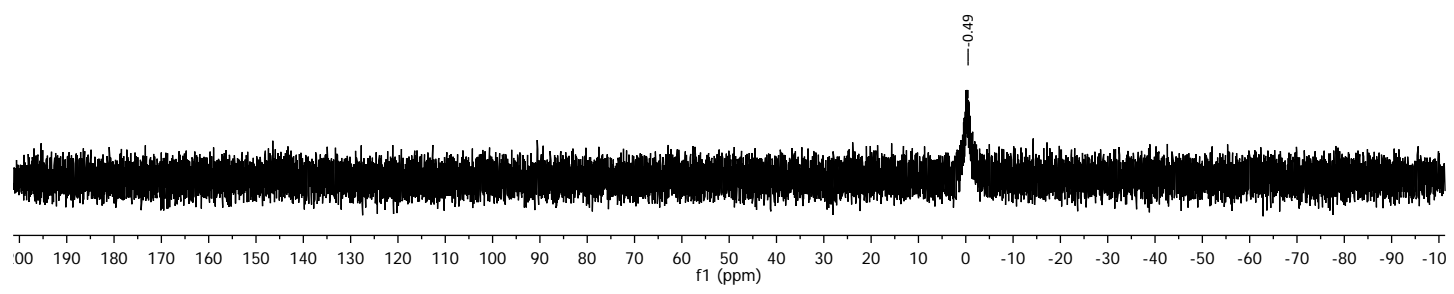

Figure S6: ${ }^{31} \mathrm{P}-\mathrm{NMR}$ spectrum $\left(\mathrm{CD}_{2} \mathrm{Cl}_{2}, 25^{\circ} \mathrm{C}, 162 \mathrm{MHz}\right)$ of 1-pAn.

\subsection{1-Piv}

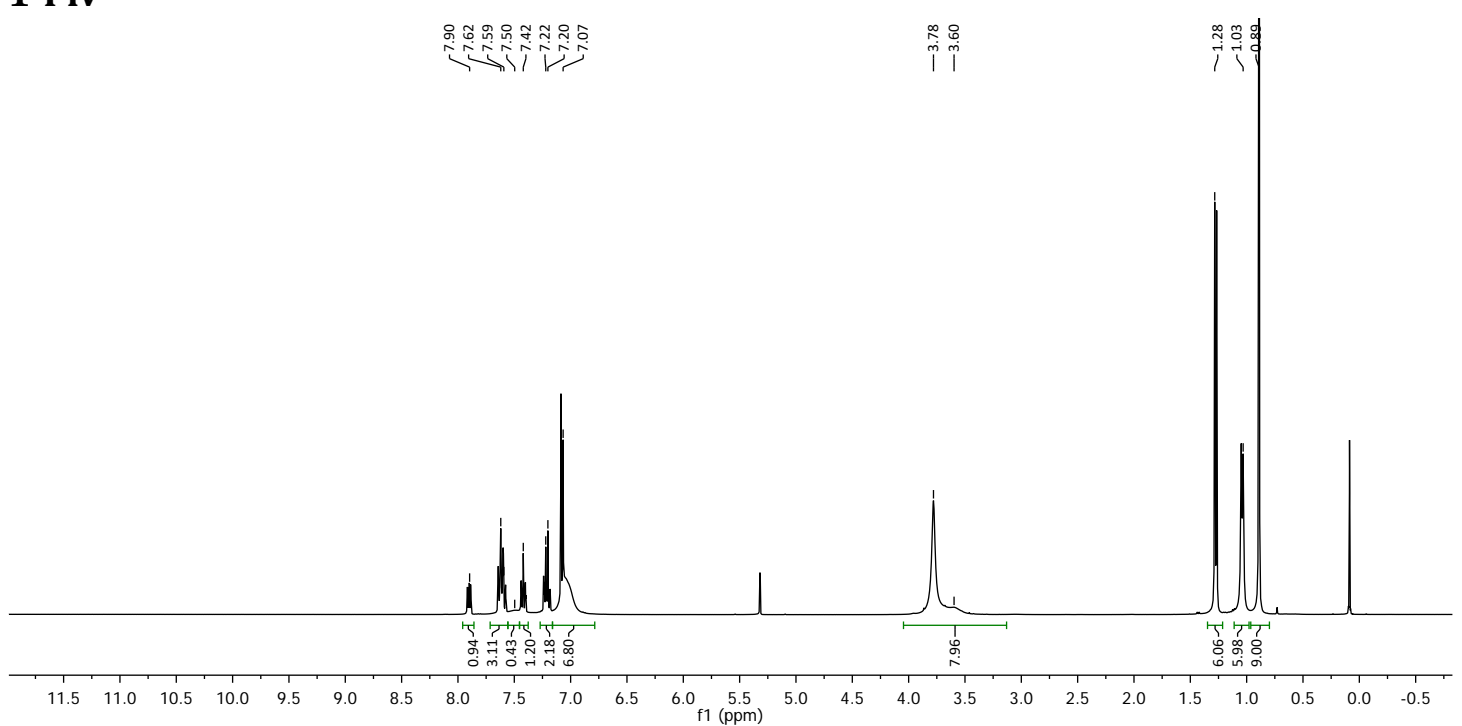

Figure S7: ${ }^{1} \mathrm{H}-\mathrm{NMR}$ spectrum $\left(\mathrm{CD}_{2} \mathrm{Cl}_{2}, 25^{\circ} \mathrm{C}, 400 \mathrm{MHz}\right)$ of 1-Piv.

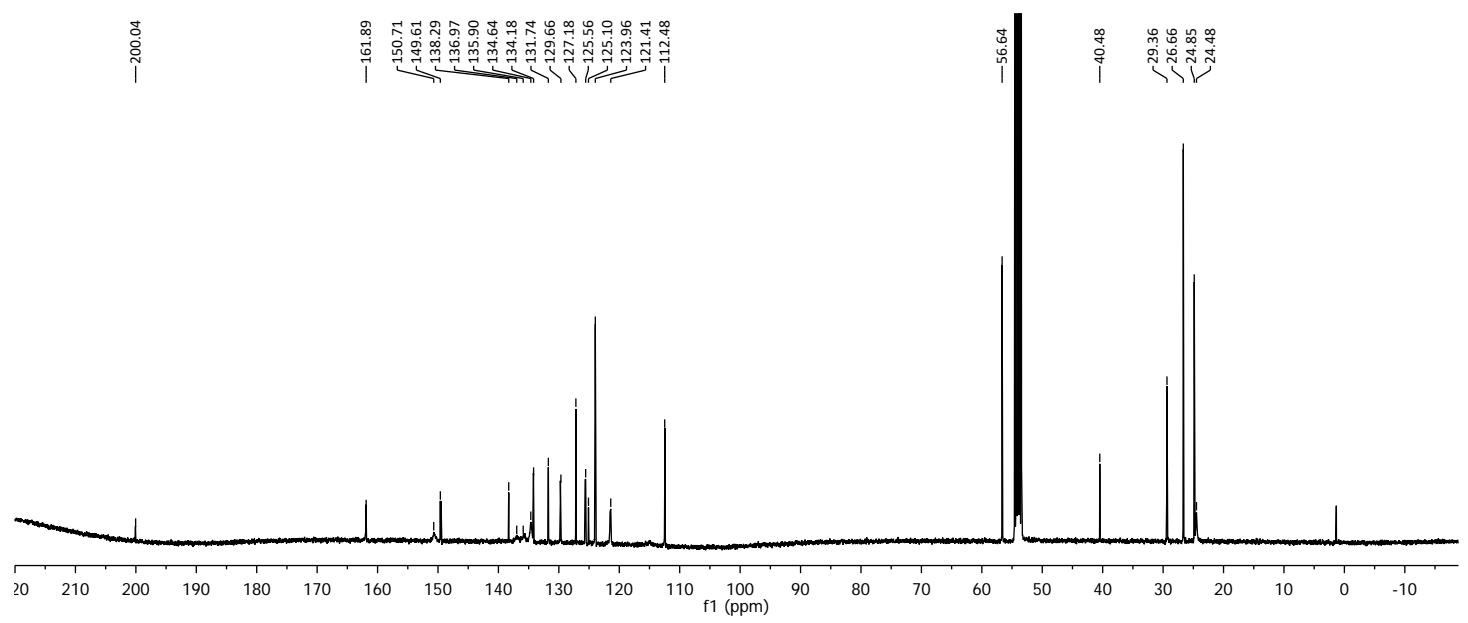

Figure S8: ${ }^{13} \mathrm{C}$-NMR spectrum $\left(\mathrm{CD}_{2} \mathrm{Cl}_{2}, 25^{\circ} \mathrm{C}, 100 \mathrm{MHz}\right)$ of 1-Piv. 


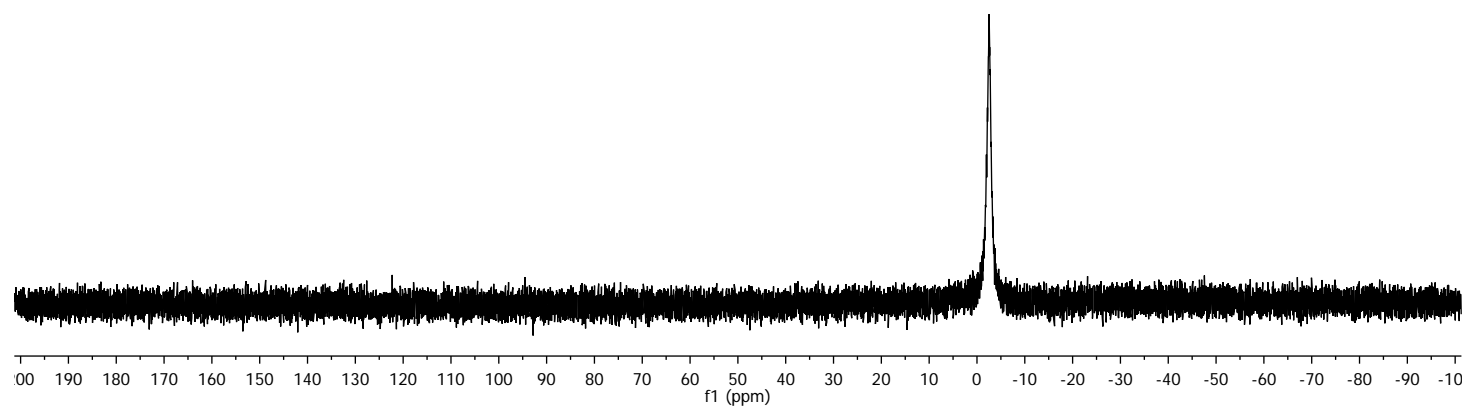

Figure S9: ${ }^{31} \mathrm{P}-\mathrm{NMR}$ spectrum $\left(\mathrm{CD}_{2} \mathrm{Cl}_{2}, 25{ }^{\circ} \mathrm{C}, 162 \mathrm{MHz}\right)$ of 1-Piv.

\subsection{1-Ph}

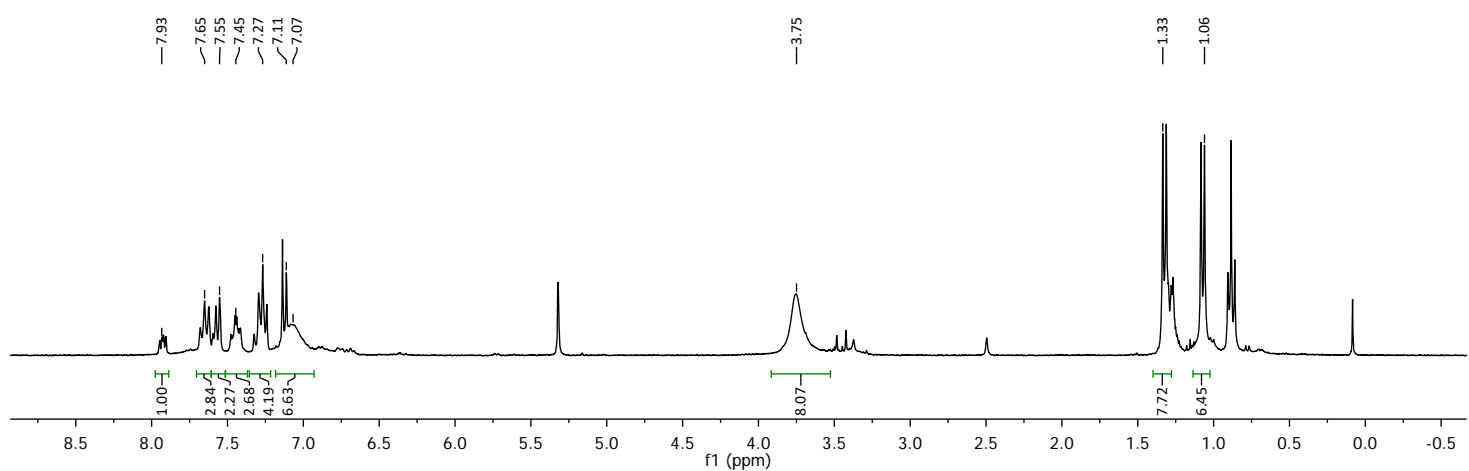

Figure S10: ${ }^{1} \mathrm{H}-\mathrm{NMR}$ spectrum $\left(\mathrm{CD}_{2} \mathrm{Cl}_{2}, 25^{\circ} \mathrm{C}, 300 \mathrm{MHz}\right)$ of 1-Ph.

\subsection{5-py}
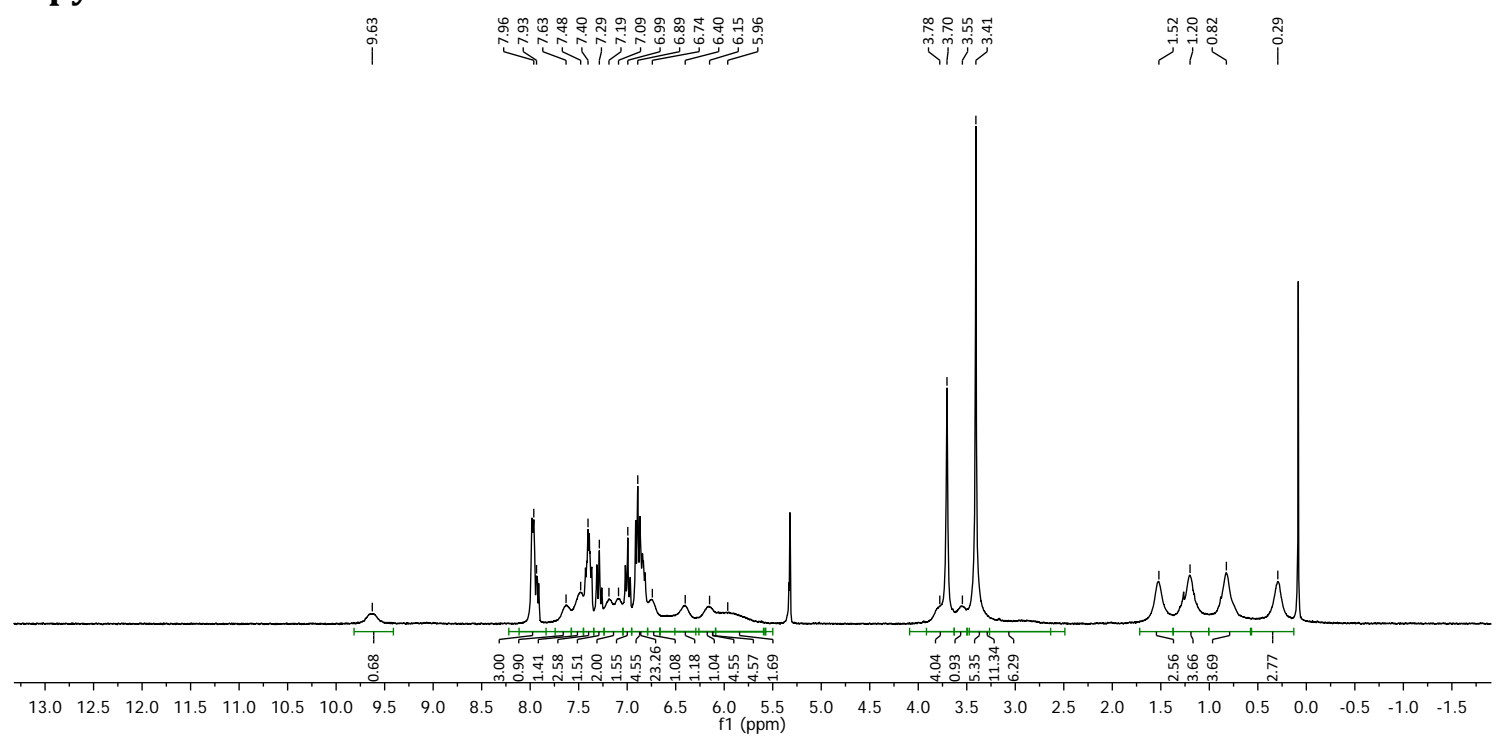

Figure S11: ${ }^{1} \mathrm{H}-\mathrm{NMR}$ spectrum $\left(\mathrm{CD}_{2} \mathrm{Cl}_{2}, 25^{\circ} \mathrm{C}, 300 \mathrm{MHz}\right)$ of 5-py. 


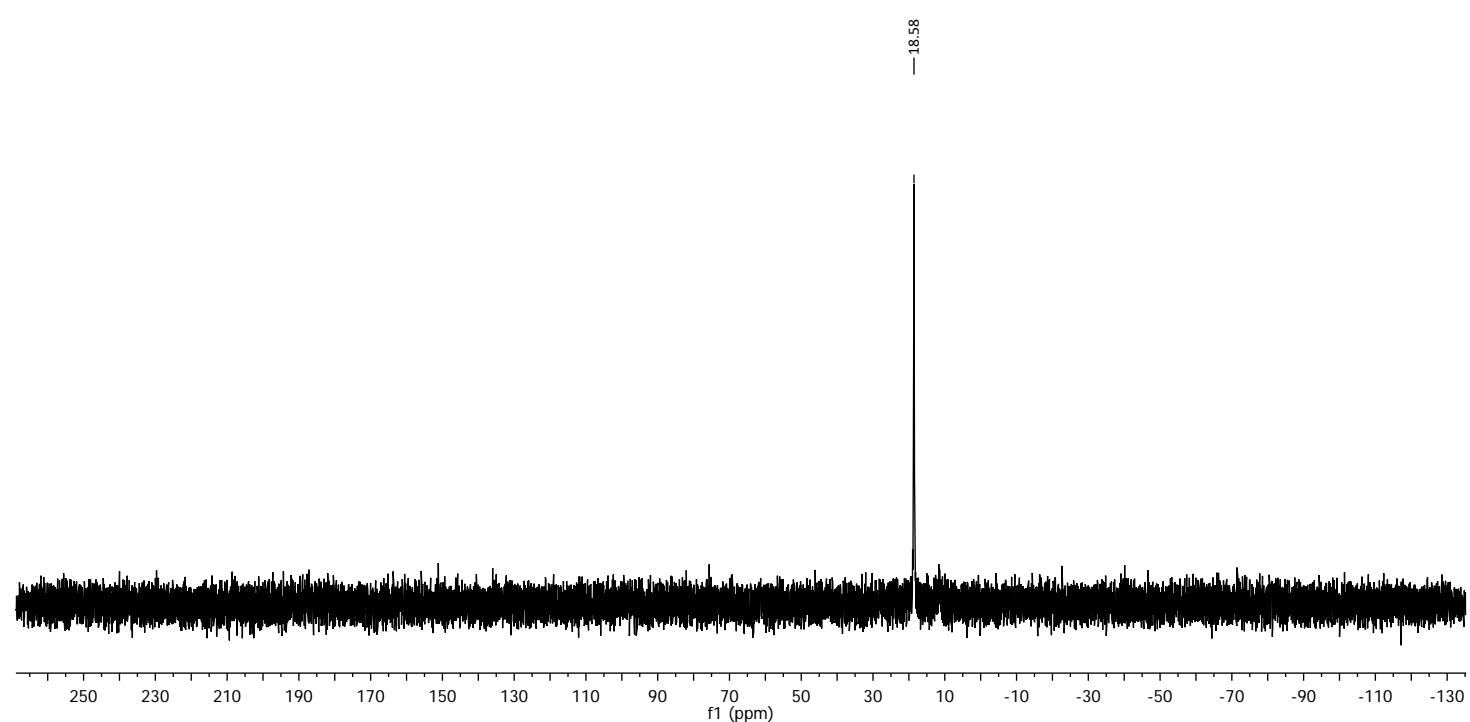

Figure S12: ${ }^{31} \mathrm{P}-\mathrm{NMR}$ spectrum $\left(\mathrm{CD}_{2} \mathrm{Cl}_{2}, 25^{\circ} \mathrm{C}, 121 \mathrm{MHz}\right)$ of 5-py.

\subsection{5-dmso}

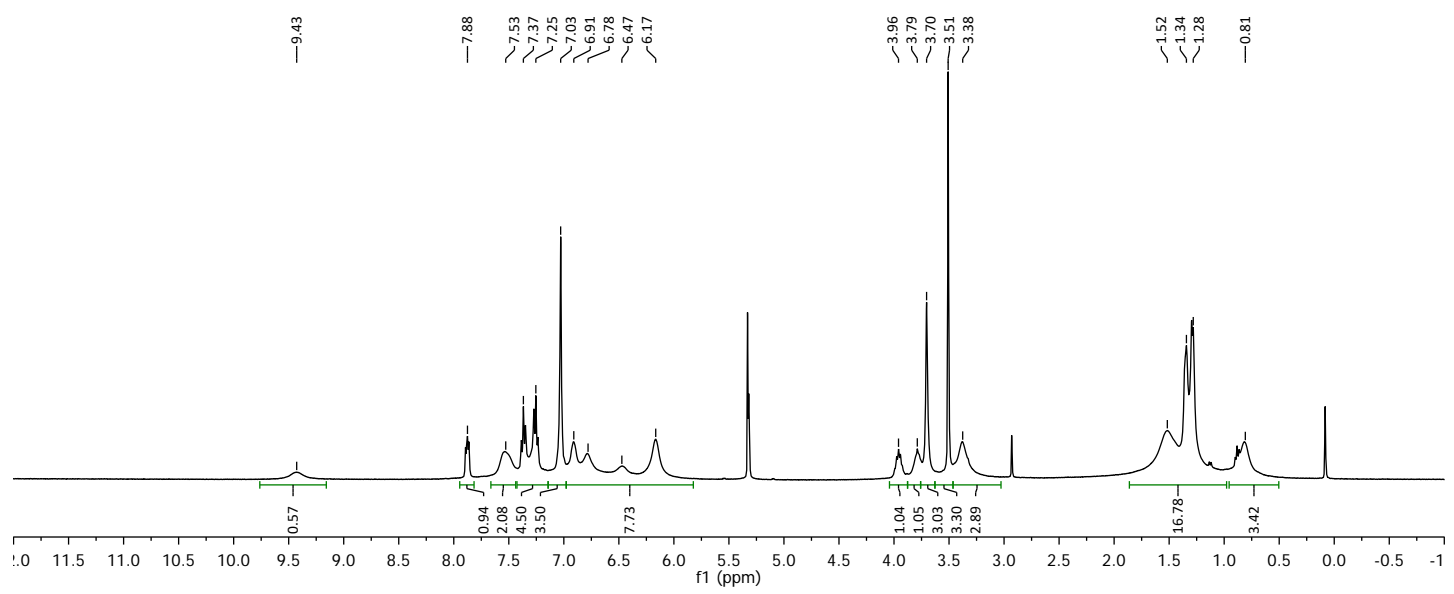

Figure S13: ${ }^{1} \mathrm{H}-\mathrm{NMR}$ spectrum $\left(\mathrm{CD}_{2} \mathrm{Cl}_{2}, 25^{\circ} \mathrm{C}, 400 \mathrm{MHz}\right)$ of 5-dmso.

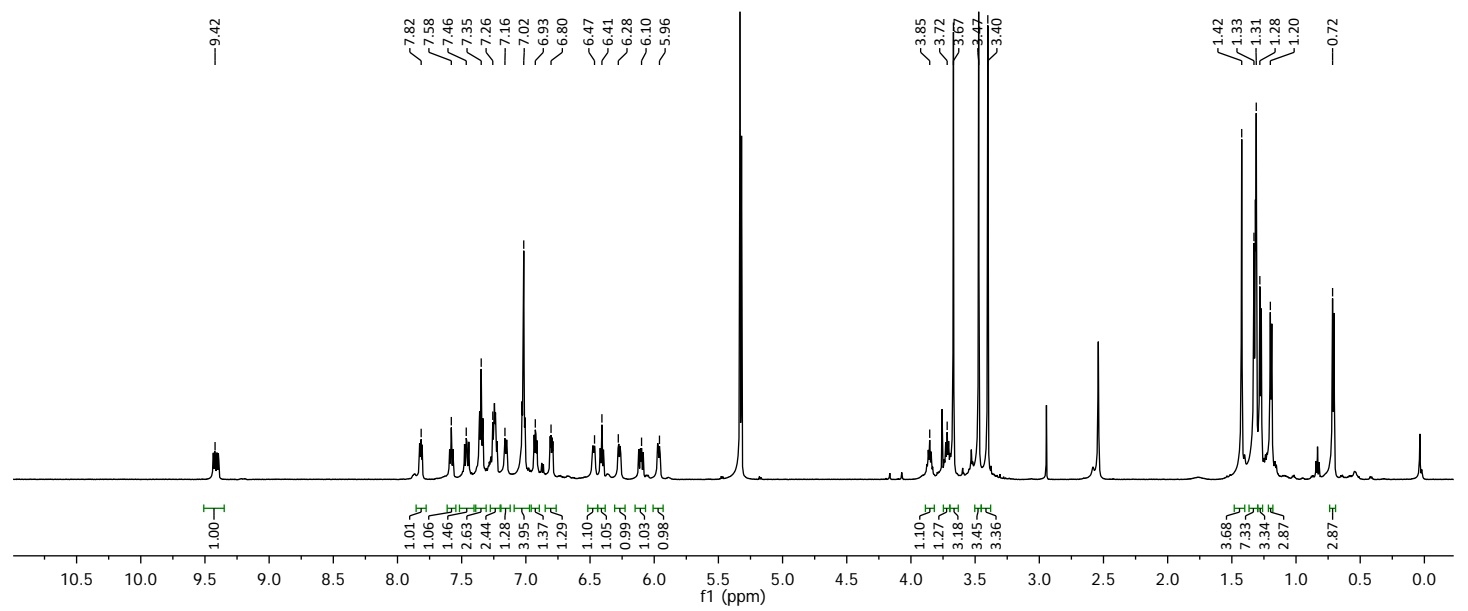

Figure S14: ${ }^{1} \mathrm{H}-\mathrm{NMR}$ spectrum $\left(\mathrm{CD}_{2} \mathrm{Cl}_{2},-30{ }^{\circ} \mathrm{C}, 600 \mathrm{MHz}\right)$ of $\mathbf{5}-\mathbf{d m s o}$. 


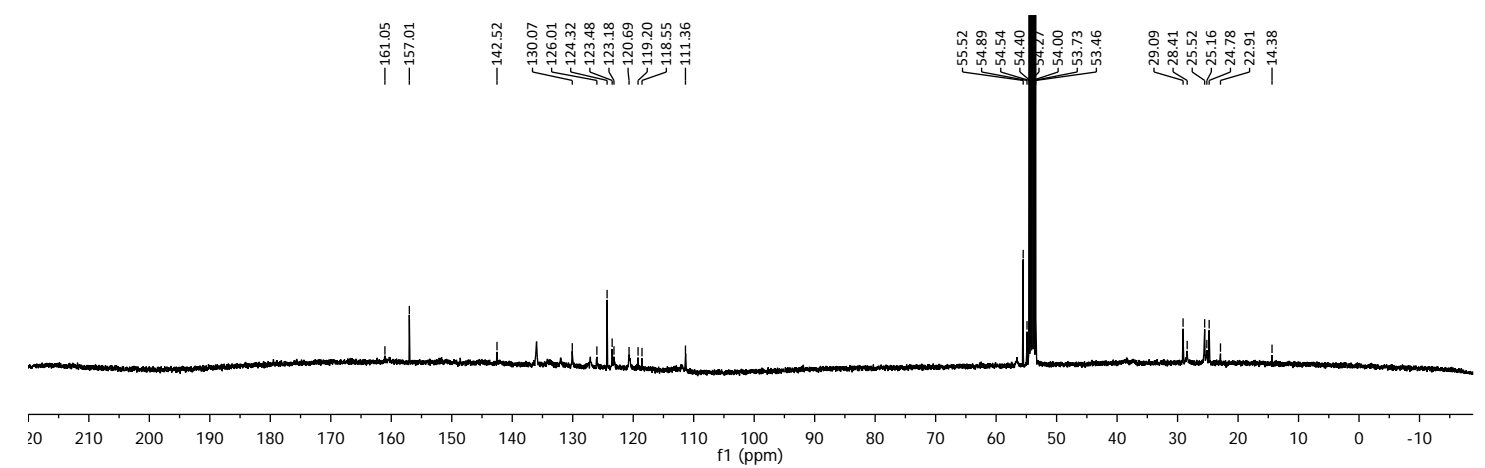

Figure S15: ${ }^{13} \mathrm{C}-\mathrm{NMR}$ spectrum $\left(\mathrm{CD}_{2} \mathrm{Cl}_{2}, 25^{\circ} \mathrm{C}, 100 \mathrm{MHz}\right)$ of $\mathbf{5}$-dmso.

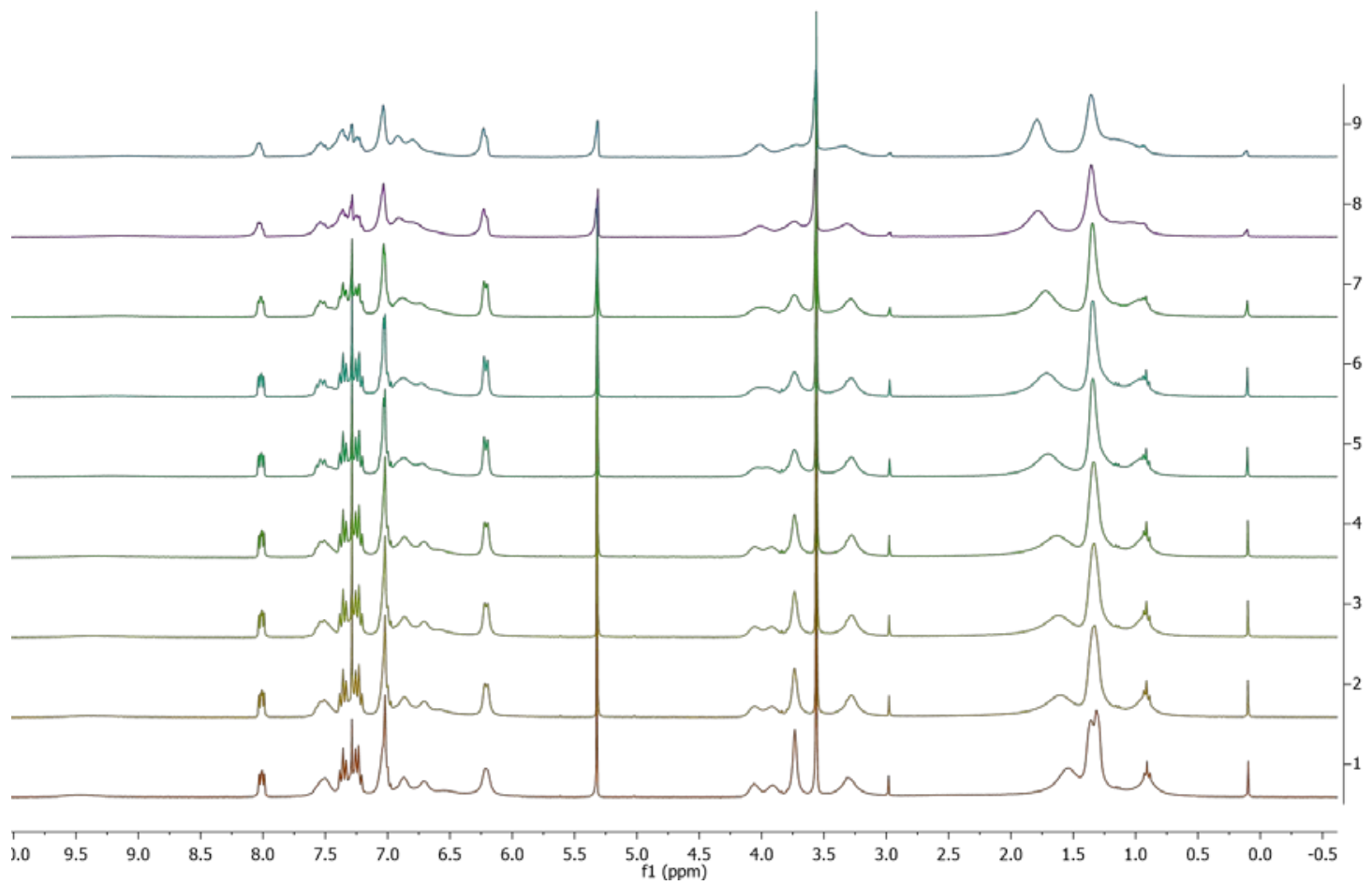

Figure S17: ${ }^{1} \mathrm{H}$-NMR spectrum $\left(\mathrm{CDCl}_{3}, 300 \mathrm{MHz}\right)$ of $\mathbf{5 - d m s o}$ at variable temperature for coalescence temperature determination. Room temperature (1), $30^{\circ} \mathrm{C}(2), 31^{\circ} \mathrm{C}(3), 32{ }^{\circ} \mathrm{C}(4), 38{ }^{\circ} \mathrm{C}(5), 39{ }^{\circ} \mathrm{C}(6), 40{ }^{\circ} \mathrm{C}$ (7) , $45^{\circ} \mathrm{C}(8), 50^{\circ} \mathrm{C}(9)$. Coalescence temperatures were determined as $\mathrm{T}_{\mathrm{c} \text {,methyl }}=31^{\circ} \mathrm{C}$ for ${ }^{\mathrm{i}} \mathrm{Pr}$-methyl signals at $1.34 \mathrm{ppm}$ and as $\mathrm{T}_{\mathrm{c} \text {, methine }}=40{ }^{\circ} \mathrm{C}$ for ${ }^{i} \mathrm{Pr}$-methine signals at $4.00 \mathrm{ppm}$. With differences in chemical shifts in the low temperature region of $\Delta v_{\text {methyl }}=15 \mathrm{~Hz}$ and $\Delta v_{\text {methyl }}=45 \mathrm{~Hz}$ an activation barrier for the chelate ring inversion process of $\Delta \mathrm{G}^{\#}=15.5 \mathrm{kcal} \mathrm{mol}^{-1}$ was determined via formulas $k_{c}=\frac{\pi \Delta v}{\sqrt{2}}$ and $\Delta G^{\#}=19.14 T_{c}(10.32+$ $\left.\log \left(\frac{T_{c}}{k_{c}}\right)\right) \cdot[1]$ 


\subsection{1,3-dimethoxy-2-(1-phenylvinyl)benzene}

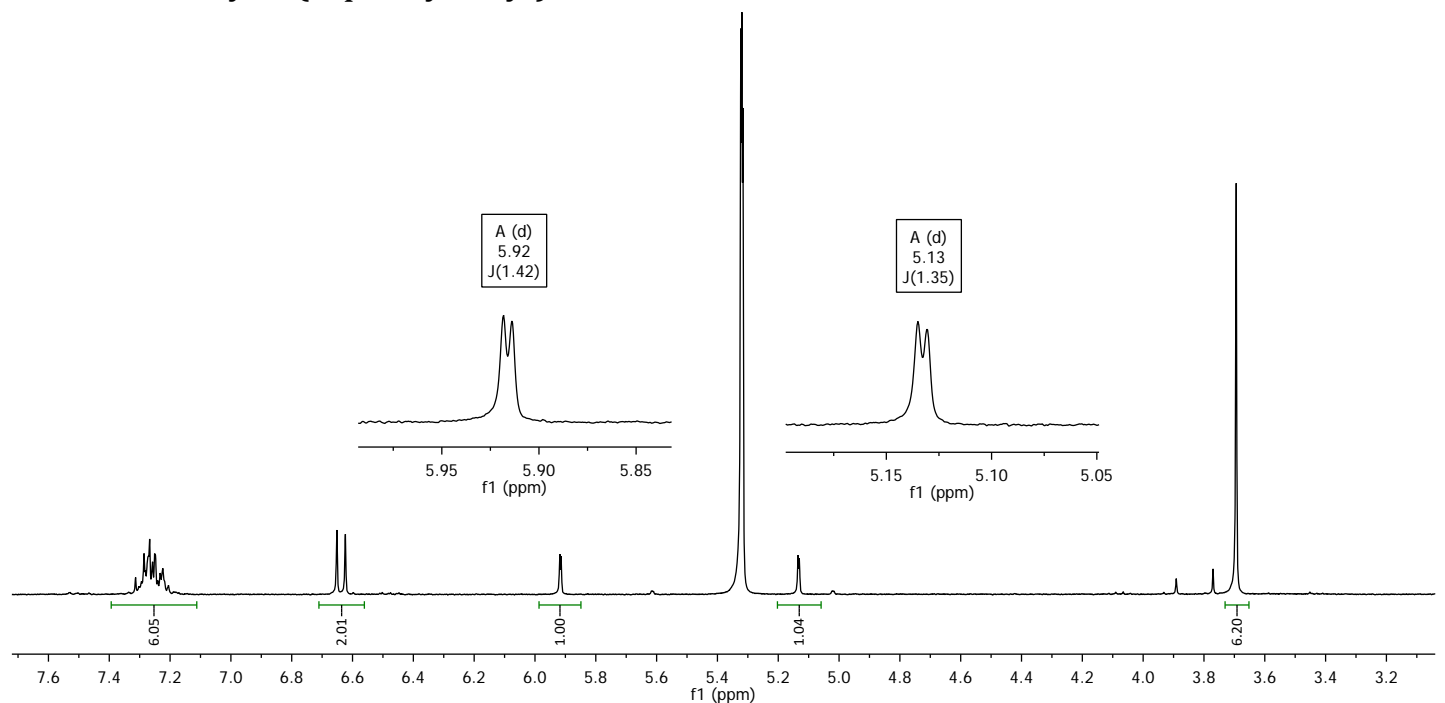

Figure S18: ${ }^{1} \mathrm{H}-\mathrm{NMR}$ spectrum $\left(\mathrm{CD}_{2} \mathrm{Cl}_{2}, 25^{\circ} \mathrm{C}, 300 \mathrm{MHz}\right)$ of the coupling product of 2,6-dimethoxy benzoic acid with styrene, 1,3-dimethoxy-2-(1-phenylvinyl)benzene.
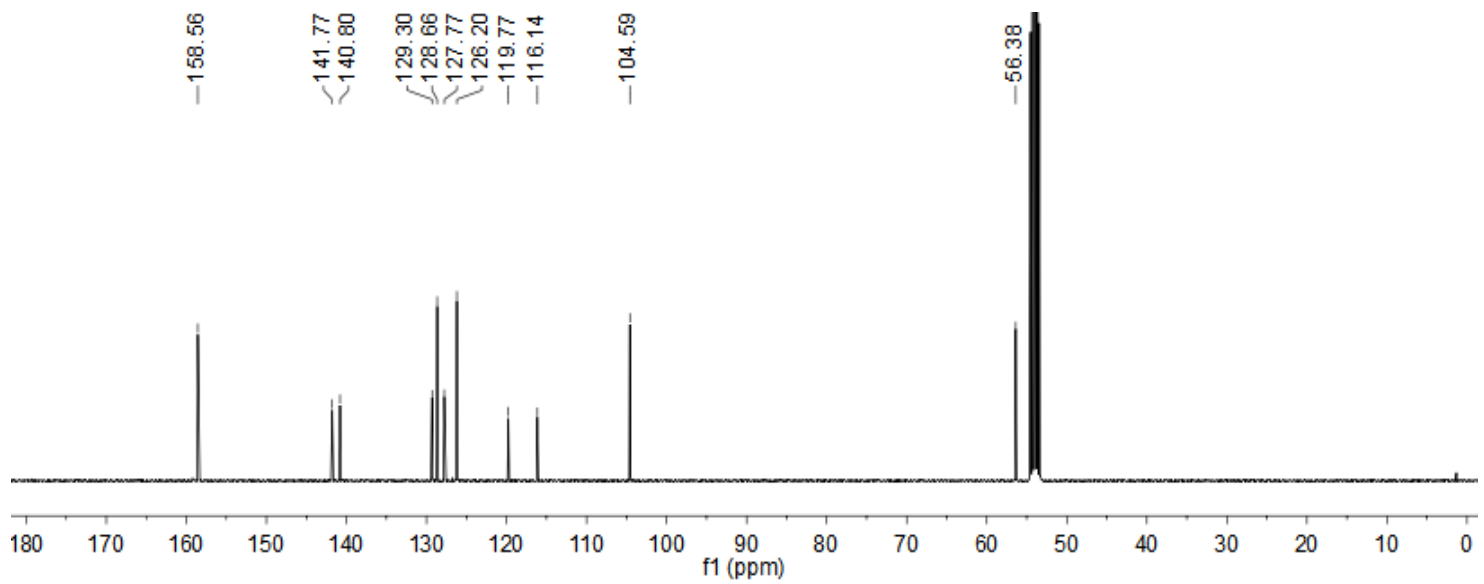

Figure S19: ${ }^{13} \mathrm{C}$-NMR spectrum $\left(\mathrm{CD}_{2} \mathrm{Cl}_{2}, 25^{\circ} \mathrm{C}, 100 \mathrm{MHz}\right)$ of the coupling product of 2,6-dimethoxy benzoic acid with styrene, 1,3-dimethoxy-2-(1-phenylvinyl)benzene. 


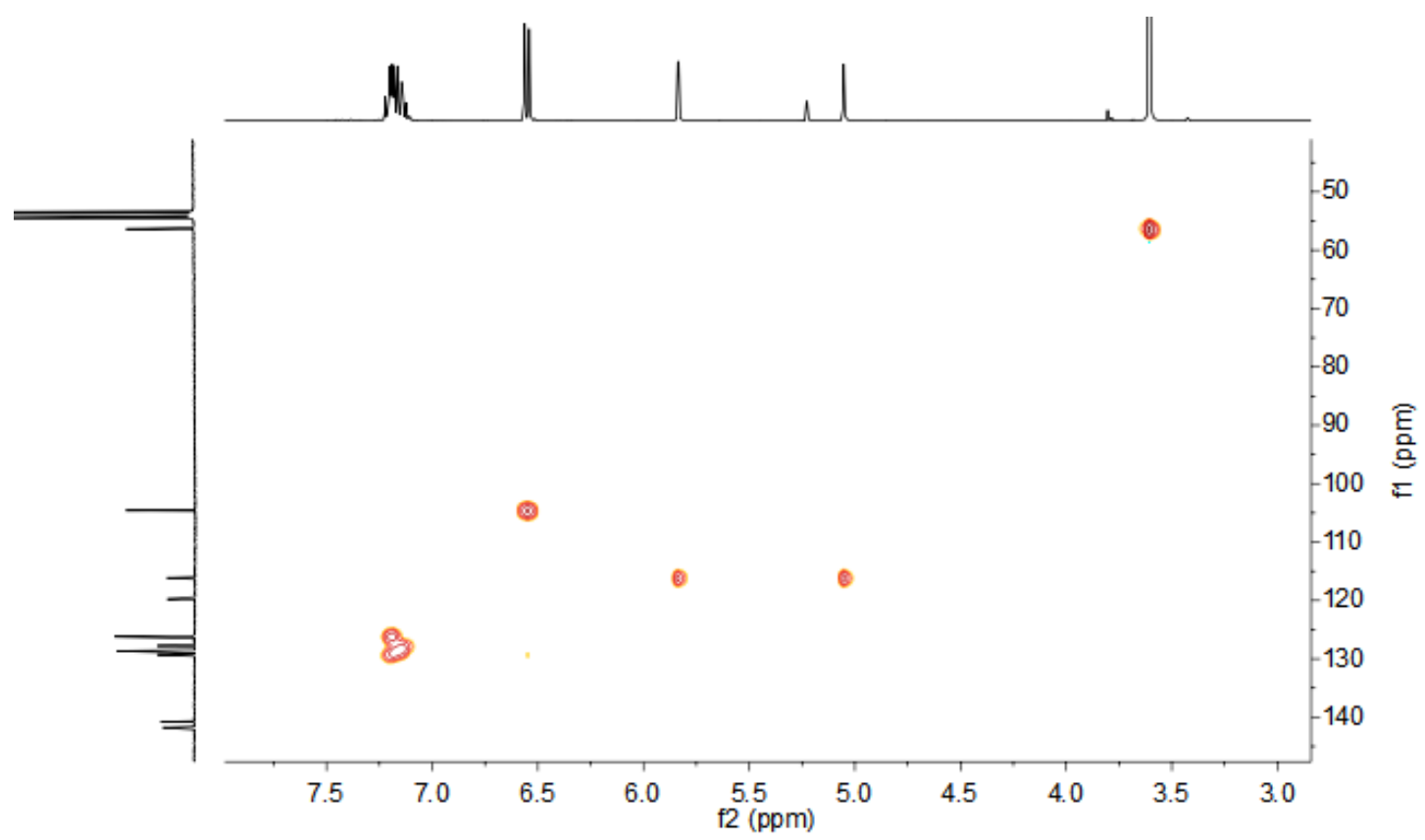

Figure S20: HSQC-NMR spectrum $\left(\mathrm{CD}_{2} \mathrm{Cl}_{2}, 25^{\circ} \mathrm{C}, 400 \mathrm{MHz}\right)$ of the coupling product of 2,6-dimethoxy benzoic acid with styrene, 1,3-dimethoxy-2-(1-phenylvinyl)benzene.

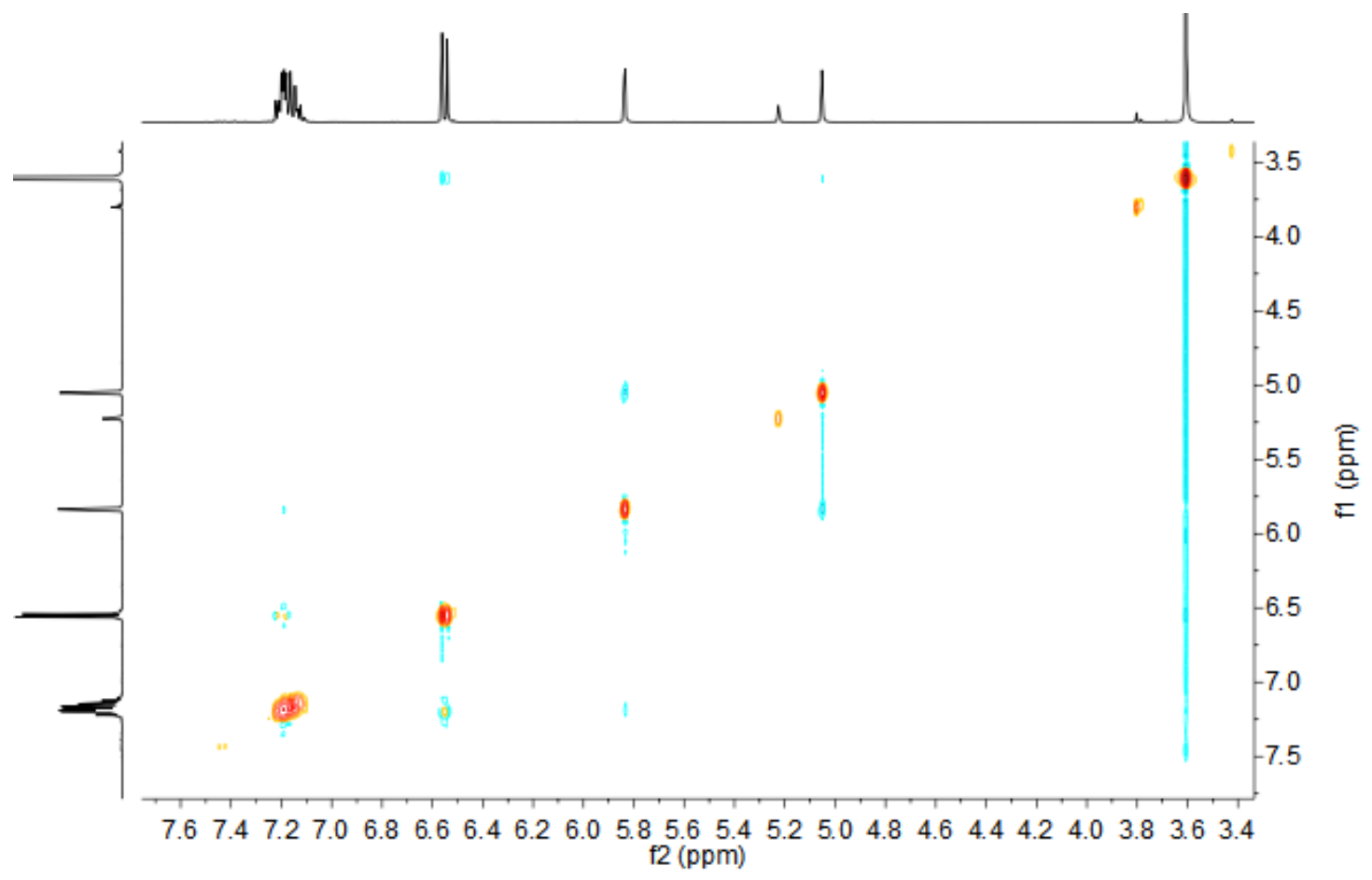

Figure S21: ${ }^{1} \mathrm{H}-1 \mathrm{H}-\mathrm{NOESY}$ spectrum $\left(\mathrm{CD}_{2} \mathrm{Cl}_{2}, 25^{\circ} \mathrm{C}, 400 \mathrm{MHz}, 0.8 \mathrm{~s}\right.$ mixing time $)$ of the coupling product of 2,6dimethoxy benzoic acid with styrene, 1,3-dimethoxy-2-(1-phenylvinyl)benzene. 


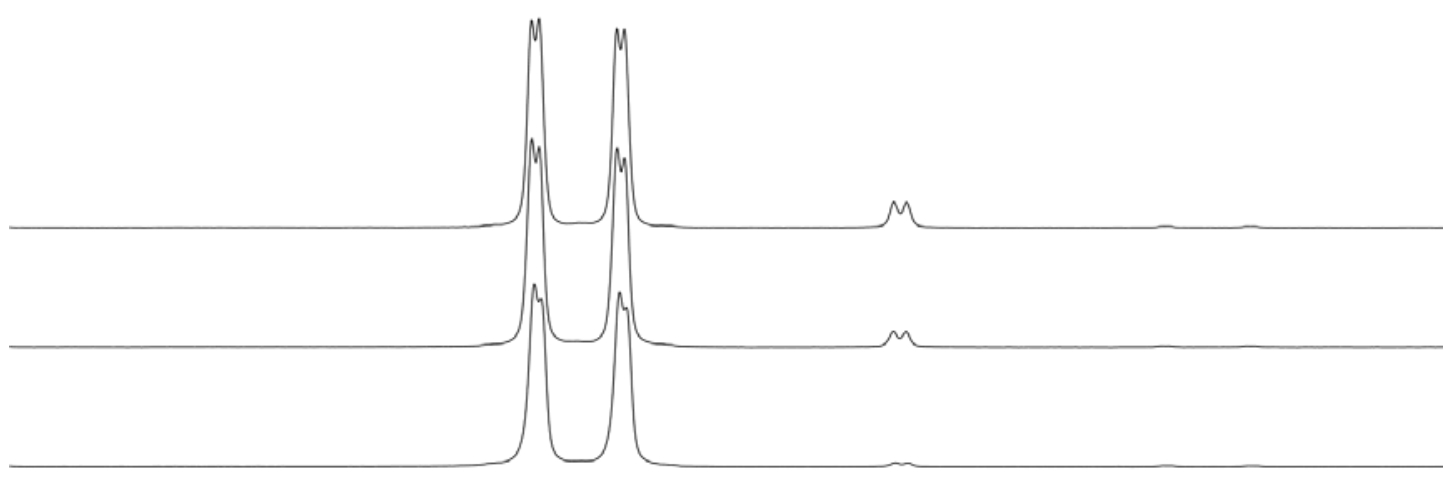

$\begin{array}{lllllllllllllllllllllllllllllll}5.42 & 5.40 & 5.38 & 5.36 & 5.34 & 5.32 & 5.30 & 5.28 & 5.26 & 5.24 & 5.22 & 5.20 & 5.18 & 5.16 & 5.14 & 5.12 & 5.10 & 5.08 & 5.06 & 5.04 & 5.02 & 5.00 & 4.98 & 4.96 & 4.94 & 4.92 & 4.90 & 4.88 & 4.86 & 4.84 & 4 .\end{array}$

Figure S22: Relevant region of the time resolved ${ }^{1} \mathrm{H}-\mathrm{NMR}$ spectrum $\left(\mathrm{THF}-d_{8}, 25^{\circ} \mathrm{C}, 300 \mathrm{MHz}\right)$ of the coupling of 2,6-dimethoxy benzoic acid with styrene $(5.19 \mathrm{ppm})$ to 1,3-dimethoxy-2-(1-phenylvinyl)benzene (5.05 ppm) with 1-Piv at room temperature after $20 \mathrm{~min}$ (bottom), $2 \mathrm{~h}$ (middle) and $2 \mathrm{~d}$ (top).

\subsection{L-Pd-Pd-L}

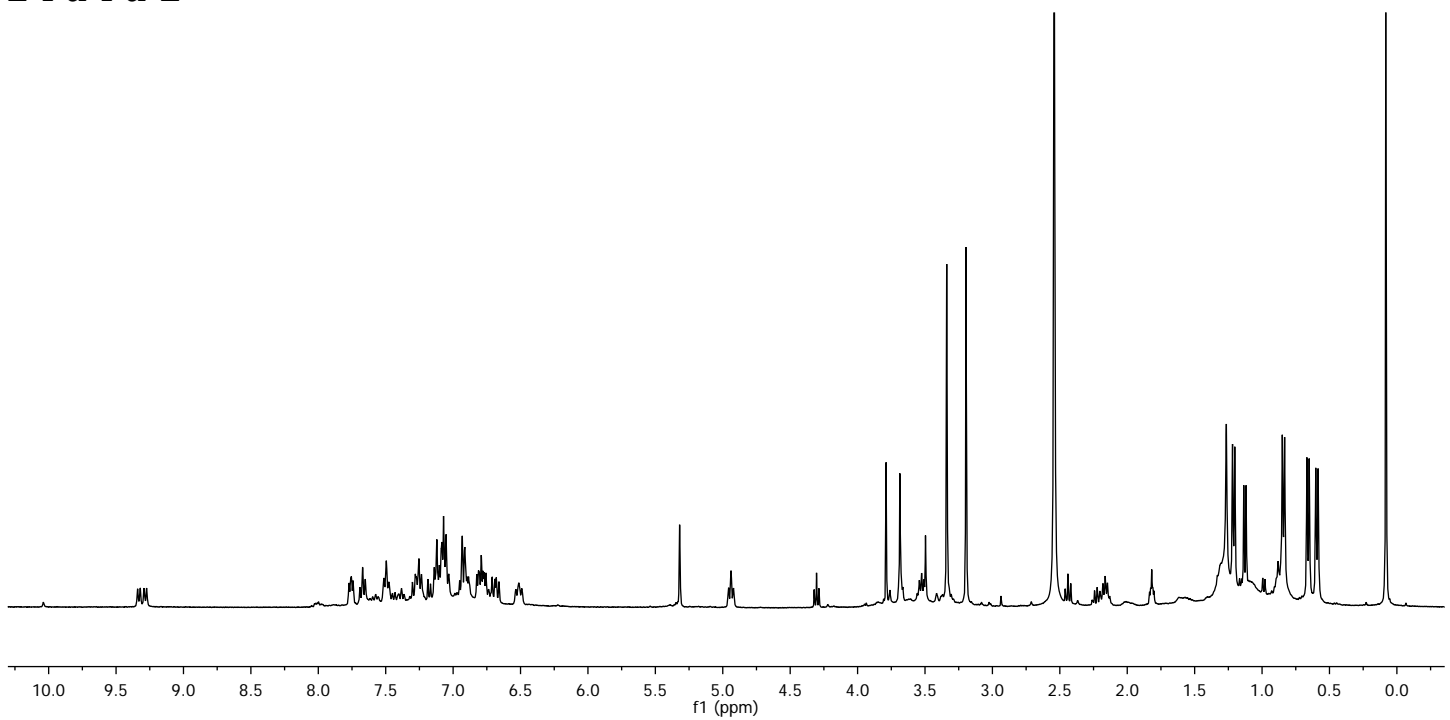

Figure S23: ${ }^{1} \mathrm{H}-\mathrm{NMR}$ spectrum $\left(\mathrm{CD}_{2} \mathrm{Cl}_{2}, 25^{\circ} \mathrm{C}, 400 \mathrm{MHz}\right)$ of the decomposition product L-Pd-Pd-L of attempted stoichiometric insertion of $\mathrm{CO}_{2}$ into 1-pAn in THF at $40^{\circ} \mathrm{C}$. Identity of this compound was confirmed via X-ray diffraction analysis. Additionally, $\mathbf{L}$ can be observed.

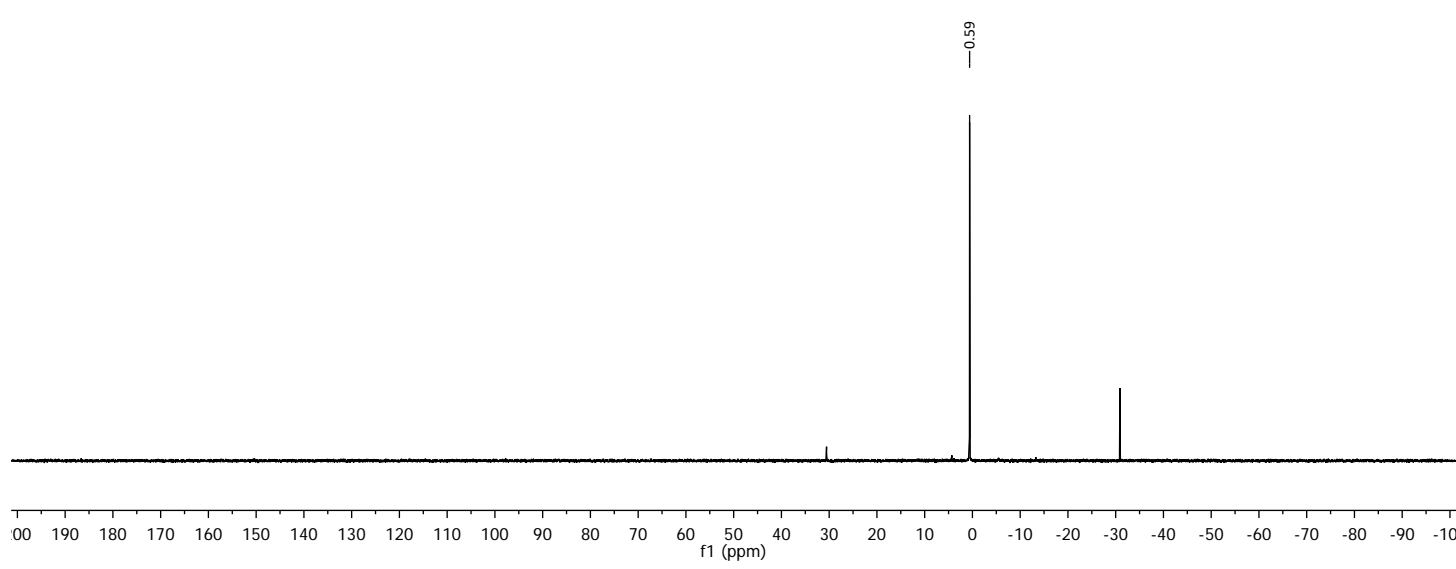

Figure S24: ${ }^{31} \mathrm{P}-\mathrm{NMR}$ spectrum $\left(\mathrm{CD}_{2} \mathrm{Cl}_{2}, 25^{\circ} \mathrm{C}, 162 \mathrm{MHz}\right)$ of the decomposition product L-Pd-Pd-L

$(0.59 \mathrm{ppm})$ of attempted stoichiometric insertion of $\mathrm{CO}_{2}$ into 1-pAn in THF at $40{ }^{\circ} \mathrm{C}$. Identity of this compound was confirmed via X-ray diffraction analysis. Additionally, L (-30.88 ppm) can be observed. 


\section{Computational Details}

The DFT-calculations in this work were performed using the GAUSSIAN 16 suite of programs (Revision B.01)[2]. The B97 density functional was employed together with the Grimme D3-dispersion correction augmented with the Becke-Johnson damping (B97-D3(BJ) $)^{[3]}$, with the automatic density fitting approximation switched on. For all atoms the def2-TZVP basis set ${ }^{[4]}$ was used and for the metal centres the corresponding ECP was applied[5]. Implicit solvent corrections were applied by using the SMD solvation model ${ }^{[6]}$ and DCM as the solvent. To account for standard state conditions a pressure of 383 atm was applied[7]. In this way all compounds were at first optimized and subsequently input to frequency calculations to characterize the nature of the stationary points to be a minimum $(i=0)$ or a transition state $(i=1)$. IRC calculations were carried out to verify that the localized transition state indeed does connect the independently localized preceding/following minima.

Table S1: DFT calculated (B97-D3(BJ)/def2-TZVP(ECP), SMD, $\mathrm{CH}_{2} \mathrm{Cl}_{2}$ ) electronic energies $E, E_{Z P E}$, enthalpies $H$, Gibbs energies $G$ and relative Gibbs energies $G_{r e l}$ of intermediates and transition states involved in the postulated catalytic carboxylation of anisole with $\mathrm{CO}_{2}$ (Scheme 1). Relative Gibbs energies refer to intermediate 1 (1-pAn).

$\begin{array}{llllll}\text { compound } & E & E_{Z P E} & H & G & G_{\text {rel }} \\ \text { anisole } & -346.6899877 & -346.560248 & -346.552235 & -346.585892 & - \\ \text { CO2 } & -188.5621065 & -188.550799 & -188.547209 & -188.565897 & - \\ \boldsymbol{p} \text {-anisic acid } & -535.2484737 & -535.103965 & -535.093175 & -535.133947 & - \\ \mathbf{1} & -2998.282928 & -2997.556719 & -2997.504134 & -2997.641262 & 0.0 \\ \mathbf{2 - A n} & -3344.975241 & -3344.117206 & -3344.056287 & -3344.20865 & 11.6 \\ \mathbf{3} & -3344.975017 & -3344.116961 & -3344.055872 & -3344.208898 & 11.5 \\ \text { TS 3-4 } & -3344.957906 & -3344.105316 & -3344.044767 & -3344.19714 & 18.8 \\ \mathbf{4} & -3344.976692 & -3344.118801 & -3344.057807 & -3344.211617 & 9.7 \\ \mathbf{5 - C O 2} & -2998.271996 & -2997.547883 & -2997.494164 & -2997.631411 & 17.4 \\ \text { TS 5-1 } & -2998.25129 & -2997.527501 & -2997.475105 & -2997.607754 & 32.2\end{array}$

Table S2: DFT calculated (B97-D3(BJ)/def2-TZVP(ECP), SMD, $\mathrm{CH}_{2} \mathrm{Cl}_{2}$ ) electronic energies $E, E_{Z P E}$, enthalpies $H$, Gibbs energies $G$ and relative Gibbs energies $G_{r e l}$ of intermediates and transition states involved in the stoichiometric insertion of $\mathrm{CO}_{2}$ into 5-dmso (Scheme 4). Relative Gibbs energies refer to 5-dmso.

$\begin{array}{llllll}\text { compound } & E & E_{Z P E} & H & G & G_{\text {rel }} \\ \text { DMSO } & -553.2319544 & -553.154333 & -553.147732 & -553.176985 & - \\ \text { CO}_{2} & -188.5621065 & -188.550799 & -188.547209 & -188.565897 & - \\ \text { 5-dmso } & -3362.955034 & -3362.163367 & -3362.10684 & -3362.249366 & 0,0 \\ \text { TS 5-1 } & -2998.25129 & -2997.527501 & -2997.475105 & -2997.607754 & 19.1 \\ \text { 1-pAn } & -2998.282928 & -2997.556719 & -2997.504134 & -2997.641262 & -1.9\end{array}$




\section{Crystallographic Data}

\subsection{5-py}

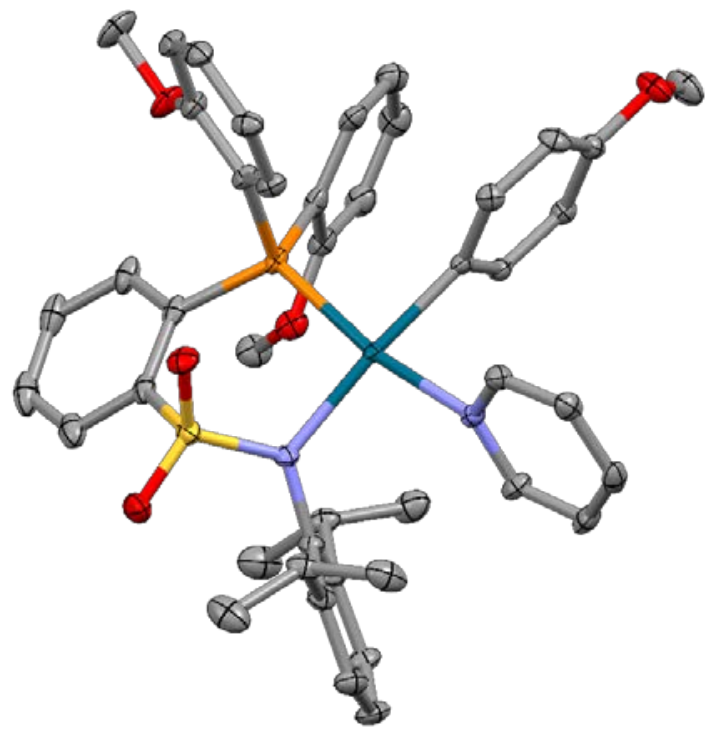

Table S3: Crystal data and structure refinement for 5-py (CCDC 2024250).

\begin{tabular}{|c|c|c|}
\hline data & 5-py & \\
\hline Identification code & 11606 & \\
\hline Empirical formula & $\mathrm{C}_{44} \mathrm{H}_{47} \mathrm{~N}_{2} \mathrm{O}_{5} \mathrm{PPdS}$ & \\
\hline Formula weight & 853.26 & \\
\hline Temperature & $100(2) \mathrm{K}$ & \\
\hline Wavelength & $0.71073 \AA$ & \\
\hline Crystal system & Monoclinic & \\
\hline Space group & $\mathrm{P} 2{ }_{1} / \mathrm{n}$, No.14 & \\
\hline \multirow[t]{3}{*}{ Unit cell dimensions } & $a=10.3054(14) \AA$ & $\alpha=90^{\circ}$ \\
\hline & $\mathrm{b}=17.978(2) \AA$ & $\beta=100.429(3)^{\circ}$. \\
\hline & $c=21.470(3) \AA$ & $\gamma=90^{\circ}$. \\
\hline Volume & $3912.0(9) \AA^{3}$ & \\
\hline $\mathrm{Z}$ & 4 & \\
\hline Density (calculated) & $1.449 \mathrm{Mg} / \mathrm{m}^{3}$ & \\
\hline Absorption coefficient & $0.617 \mathrm{~mm}^{-1}$ & \\
\hline$F(000)$ & 1768 & \\
\hline Crystal size & $0.086 \times 0.031 \times 0.022 \mathrm{~mm}^{3}$ & \\
\hline Theta range for data collection & 2.266 to $34.490^{\circ}$ & \\
\hline Index ranges & $\begin{array}{l}-16<=\mathrm{h}<=16,-28<=\mathrm{k}<=28,- \\
34<=\mathrm{l}<=34\end{array}$ & \\
\hline Reflections collected & 139594 & \\
\hline Independent reflections & $16490[\mathrm{R}(\mathrm{int})=0.0921]$ & \\
\hline $\begin{array}{l}\text { Completeness to theta }= \\
25.242^{\circ}\end{array}$ & $99.9 \%$ & \\
\hline Absorption correction & Gaussian & \\
\hline Max. and min. transmission & 0.98843 and 0.96604 & \\
\hline Refinement method & Full-matrix least-squares on $\mathrm{F}^{2}$ & \\
\hline Data / restraints / parameters & $16490 / 0$ / 494 & \\
\hline Goodness-of-fit on $\mathrm{F}^{2}$ & 1.032 & \\
\hline Final R indices [I>2sigma(I)] & $\mathrm{R} 1=0.0407, \mathrm{wR} 2=0.0780$ & \\
\hline $\mathrm{R}$ indices (all data) & $\mathrm{R} 1=0.0727, \mathrm{wR} 2=0.0890$ & \\
\hline Extinction coefficient & $\mathrm{n} / \mathrm{a}$ & \\
\hline Largest diff. peak and hole & 0.945 and -1.135 e. $\AA^{-3}$ & \\
\hline
\end{tabular}




\subsection{5-dmso}

Comment: This compound crystallized in the composition $0.33^{*} \mathbf{5}-\mathbf{d m s o}+0.67 * \mathbf{5}$-py.

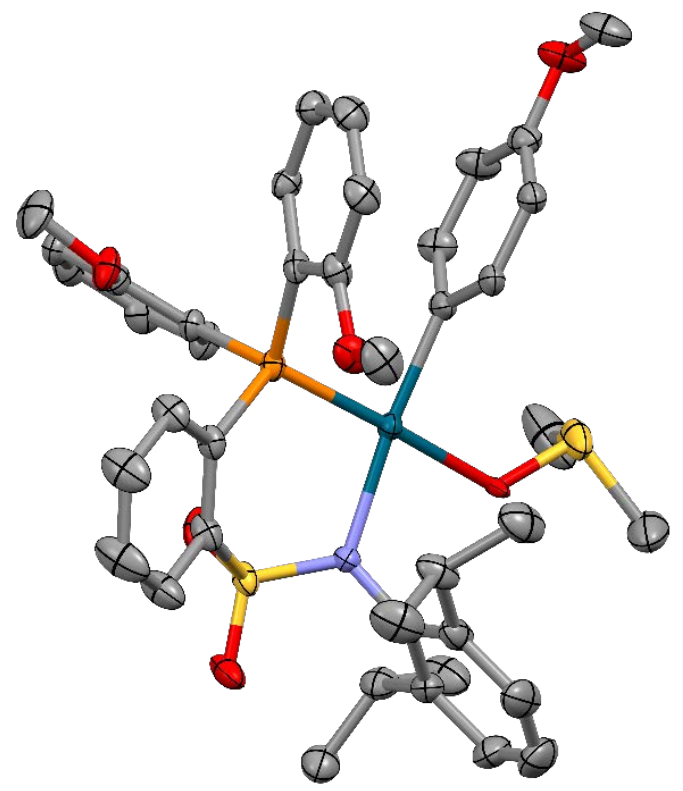

Table S4: Crystal data and structure refinement for 5-dmso (CCDC 2024251).

\begin{tabular}{|c|c|c|}
\hline data & 5-dmso & \\
\hline Identification code & 11665 & \\
\hline Empirical formula & $\mathrm{C}_{42.99} \mathrm{H}_{45.33} \mathrm{~N}_{1.66} \mathrm{O}_{5.34} \mathrm{PPdS}_{1.34}$ & \\
\hline Formula weight & 850.92 & \\
\hline Temperature & $100(2) \mathrm{K}$ & \\
\hline Wavelength & $0.71073 \AA ̊$ & \\
\hline Crystal system & Monoclinic & \\
\hline Space group & P $21_{1} / \mathrm{n}$, No. 14 & \\
\hline \multirow[t]{3}{*}{ Unit cell dimensions } & $a=10.4283(12) \AA$ & $\alpha=90^{\circ}$ \\
\hline & $\mathrm{b}=19.554(2) \AA$ & $\beta=101.601(2)^{\circ}$. \\
\hline & $c=19.851(2) \AA$ & $\gamma=90^{\circ}$ \\
\hline Volume & $3965.2(7) \AA 3$ & \\
\hline $\mathrm{Z}$ & 4 & \\
\hline Density (calculated) & $1.425 \mathrm{Mg} / \mathrm{m} 3$ & \\
\hline Absorption coefficient & $0.626 \mathrm{~mm}-1$ & \\
\hline$F(000)$ & 1760 & \\
\hline Crystal size & $0.066 \times 0.043 \times 0.018 \mathrm{~mm} 3$ & \\
\hline Theta range for data collection & 2.057 to $30.706^{\circ}$ & \\
\hline Index ranges & $-14<=\mathrm{h}<=14,-27<=\mathrm{k}<=27,-$ & \\
\hline & $28<=\mathrm{l}<=28$ & \\
\hline Reflections collected & 113775 & \\
\hline Independent reflections & $12169[\mathrm{R}(\mathrm{int})=0.1178]$ & \\
\hline $\begin{array}{l}\text { Completeness to theta }= \\
25.242^{\circ}\end{array}$ & $99.9 \%$ & \\
\hline Absorption correction & Gaussian & \\
\hline Max. and min. transmission & 0.99040 and 0.96509 & \\
\hline Refinement method & Full-matrix least-squares on $\mathrm{F}^{2}$ & \\
\hline Data / restraints / parameters & 12169 / 40 / 501 & \\
\hline Goodness-of-fit on $\mathrm{F}^{2}$ & 1.022 & \\
\hline Final R indices [I>2sigma(I)] & $\mathrm{R} 1=0.0482, \mathrm{wR} 2=0.0943$ & \\
\hline $\mathrm{R}$ indices (all data) & $\mathrm{R} 1=0.0894, \mathrm{wR} 2=0.1116$ & \\
\hline Extinction coefficient & $\mathrm{n} / \mathrm{a}$ & \\
\hline Largest diff. peak and hole & 1.138 and -0.805 e. $. \AA-3$ & \\
\hline
\end{tabular}




\subsection{1-pAn}

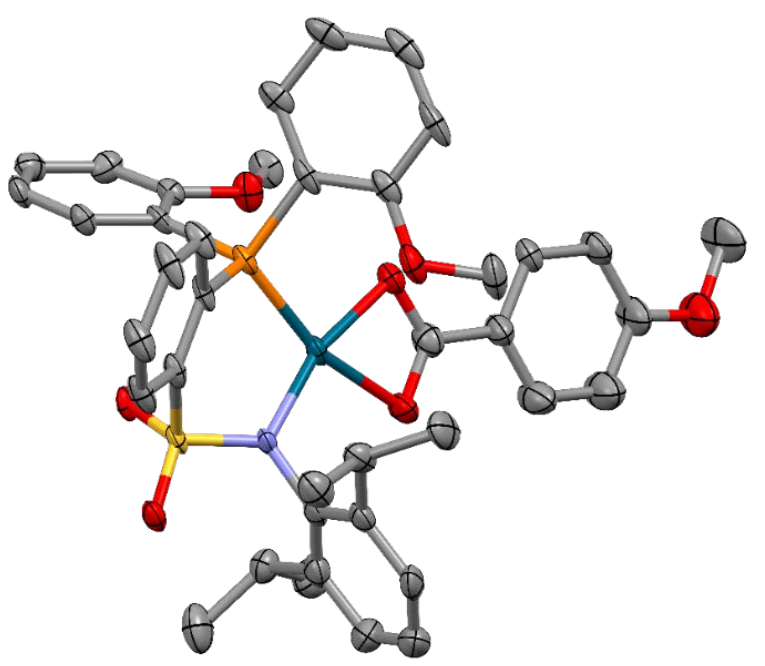

Table S5: Crystal data and structure refinement for 1-pAn (CCDC 2042213).

\begin{tabular}{|c|c|c|}
\hline data & 1-pAn & \\
\hline Identification code & $12624 S Q$ & \\
\hline Empirical formula & C40 H42 N 07 P Pd S & \\
\hline Formula weight & 818.17 & \\
\hline Temperature & $100(2) \mathrm{K}$ & \\
\hline Wavelength & $0.71073 \AA$ & \\
\hline Crystal system & Trigonal & \\
\hline Space group & $\mathrm{R}-3$ & \\
\hline \multirow[t]{3}{*}{ Unit cell dimensions } & $a=54.663(8) \AA$ & $\alpha=90^{\circ}$ \\
\hline & $\mathrm{b}=54.663(8) \AA$ & $\beta=90^{\circ}$. \\
\hline & $c=10.056(2) \AA$ & $\gamma=120^{\circ}$. \\
\hline Volume & $26022(9) \AA^{3}$ & \\
\hline $\mathrm{Z}$ & 18 & \\
\hline Density (calculated) & $0.940 \mathrm{Mg} / \mathrm{m}^{3}$ & \\
\hline Absorption coefficient & $0.417 \mathrm{~mm}^{-1}$ & \\
\hline $\mathrm{F}(000)$ & 7596 & \\
\hline Crystal size & $0.190 \times 0.060 \times 0.050 \mathrm{~mm}^{3}$ & \\
\hline Theta range for data collection & 2.658 to $26.000^{\circ}$. & \\
\hline Index ranges & $-67<=\mathrm{h}<=67,-67<=\mathrm{k}<=67,-12<=\mathrm{l}<=12$ & \\
\hline Reflections collected & 61892 & \\
\hline Independent reflections & $10885[\mathrm{R}(\mathrm{int})=0.1221]$ & \\
\hline Completeness to theta $=25.242^{\circ}$ & $95.5 \%$ & \\
\hline Absorption correction & Gaussian & \\
\hline Max. and min. transmission & 0.97821 and 0.93879 & \\
\hline Refinement method & Full-matrix least-squares on $\mathrm{F}^{2}$ & \\
\hline Data / restraints / parameters & 10885 / 99 / 467 & \\
\hline Goodness-of-fit on $\mathrm{F}^{2}$ & 1.079 & \\
\hline Final $R$ indices $[\mathrm{I}>2 \operatorname{sigma}(\mathrm{I})]$ & $\mathrm{R} 1=0.0939, \mathrm{wR} 2=0.2497$ & \\
\hline $\mathrm{R}$ indices (all data) & $\mathrm{R} 1=0.1228, \mathrm{wR} 2=0.2817$ & \\
\hline Extinction coefficient & $\mathrm{n} / \mathrm{a}$ & \\
\hline Largest diff. peak and hole & 2.752 and -4.520 e. $\AA^{-3}$ & \\
\hline
\end{tabular}

The low R1 value of this structure is attributed to a changing quality of the crystal during X-ray data acquisition, which in turn is a result of volatile solvent molecules evaporating from the crystal. Determination of atom connecitivities, however, is valid. 


\subsection{1-Ph}

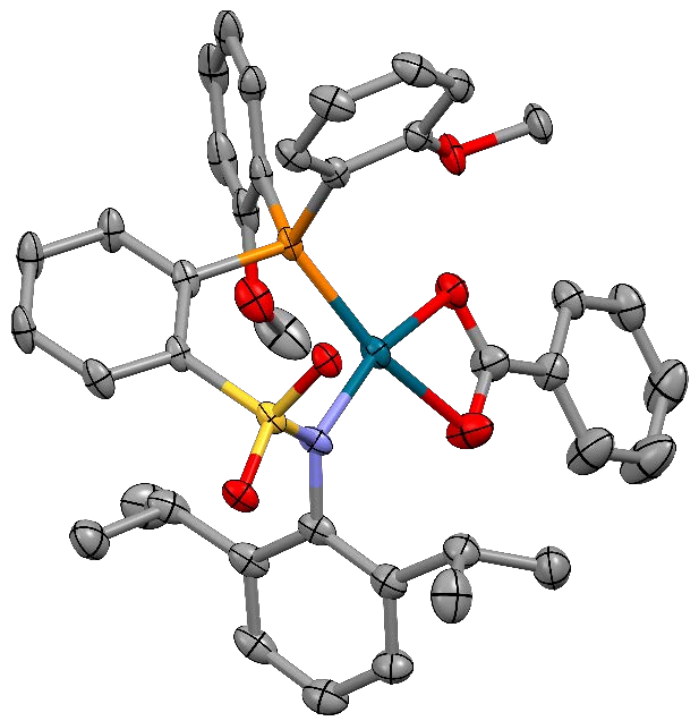

Table S6: Crystal data and structure refinement for 1-Ph (CCDC 2024252).

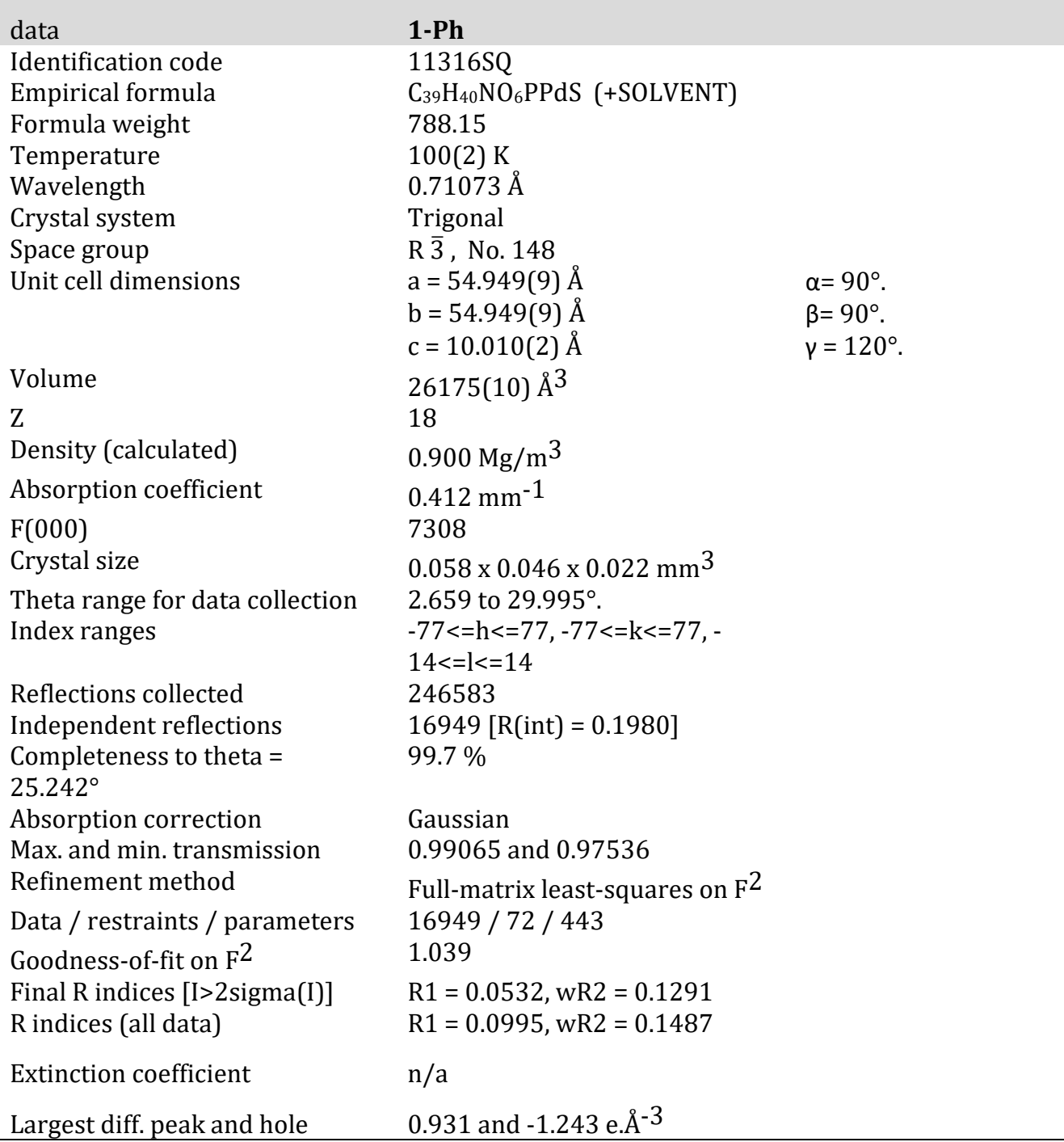




\section{References}

[1] H. Friebolin, J. K. Becconsall, Basic one-and two-dimensional NMR spectroscopy, VCH Weinheim, 1993.

[2] Gaussian 16, Revision B.01, M. J. Frisch, G. W. Trucks, H. B. Schlegel, G. E. Scuseria, M. A. Robb, J. R. Cheeseman, G. Scalmani, V. Barone, G. A. Petersson, H. Nakatsuji, X. Li, M. Caricato, A. V. Marenich, J. Bloino, B. G. Janesko, R. Gomperts, B. Mennucci, H. P. Hratchian, J. V. Ortiz, A. F. Izmaylov, J. L. Sonnenberg, D. Williams-Young, F. Ding, F. Lipparini, F. Egidi, J. Goings, B. Peng, A. Petrone, T. Henderson, D. Ranasinghe, V. G. Zakrzewski, J. Gao, N. Rega, G. Zheng, W. Liang, M. Hada, M. Ehara, K. Toyota, R. Fukuda, J. Hasegawa, M. Ishida, T. Nakajima, Y. Honda, O. Kitao, H. Nakai, T. Vreven, K. Throssell, J. A. Montgomery, Jr., J. E. Peralta, F. Ogliaro, M. J. Bearpark, J. J. Heyd, E. N. Brothers, K. N. Kudin, V. N. Staroverov, T. A. Keith, R. Kobayashi, J. Normand, K. Raghavachari, A. P. Rendell, J. C. Burant, S. S. Iyengar, J. Tomasi, M. Cossi, J. M. Millam, M. Klene, C. Adamo, R. Cammi, J. W. Ochterski, R. L. Martin, K. Morokuma, O. Farkas, J. B. Foresman, and D. J. Fox, Gaussian, Inc., Wallingford CT, 2016.

[3] a) S. Grimme, Journal of computational chemistry 2004, 25, 1463-1473; b) J. Antony, S. Grimme, Physical Chemistry Chemical Physics 2006, 8, 5287-5293; c) S. Grimme, Journal of computational chemistry 2006, 27, 1787-1799; d) M. Piacenza, I. Hyla-Kryspin, S. Grimme, Journal of computational chemistry 2007, 28, 2275-2285; e) S. Grimme, J. Antony, S. Ehrlich, H. Krieg, The Journal of chemical physics 2010, 132, 154104; f) S. Grimme, S. Ehrlich, L. Goerigk, Journal of computational chemistry 2011, 32, 1456-1465.

[4] a) A. Schäfer, H. Horn, R. Ahlrichs, The Journal of Chemical Physics 1992, 97, 2571-2577; b) A. Schäfer, C. Huber, R. Ahlrichs, The Journal of Chemical Physics 1994, 100, 5829-5835; c) K. Eichkorn, F. Weigend, 0. Treutler, R. Ahlrichs, Theoretical Chemistry Accounts 1997, 97, 119-124; d) F. Weigend, F. Furche, R. Ahlrichs, The Journal of chemical physics 2003, 119, 12753-12762; e) F. Weigend, R. Ahlrichs, Physical Chemistry Chemical Physics 2005, 7, 3297-3305.

[5] a) D. Andrae, U. Haeussermann, M. Dolg, H. Stoll, H. Preuss, Theoretica chimica acta 1990, 77, 123-141;

b) B. Metz, H. Stoll, M. Dolg, The Journal of Chemical Physics 2000, 113, 2563-2569.

[6] a) E. Cances, B. Mennucci, J. Tomasi, The Journal of chemical physics 1997, 107, 3032-3041; b) J. Tomasi, B. Mennucci, R. Cammi, Chemical reviews 2005, 105, 2999-3094; c) A. V. Marenich, C. J. Cramer, D. G. Truhlar, The Journal of Physical Chemistry B 2009, 113, 6378-6396.

[7] R. L. Martin, P. J. Hay, L. R. Pratt, The Journal of Physical Chemistry A 1998, 102, 3565-3573. 